\title{
ORIENTACIONES EDUCATIVAS PARA PROMOVER EL CRECIMIENTO PERSONAL EN LOS JÓVENES. UNA PROPUESTA PARA EDUCADORES
}

\author{
Educational Guidelines to Promote Personal Growth in Young People. A Proposal \\ for Educators
}

\author{
Juana María Cubela González, Dra. C. \\ Universidad de Oriente, Cuba \\ https://orcid.org/0000-0002-1585-6536 \\ jmcubela@uo.edu.cu
}

Palabras claves: Crecimiento Personal, Orientación Educativa, Educadores.

Keywords: Personal Growth, Educational Orientation, Educators.
Recibido: 05 de marzo de 2021

Aceptado: 28 de abril de 2021

\section{RESUMEN}

Los educadores, con responsabilidad de influir positivamente en la formación integral de la juventud, tienen ante sí la compleja tarea de contribuir al desarrollo y crecimiento personal de los jóvenes, han de tener a su alcance alternativas que le permitan conducir, con intencionalidad, esta complicada responsabilidad. Es propósito de la autora sugerir vías, acciones de orientación educativa, procedimientos que contribuyan a potenciar en la juventud la capacidad de asumir su propio crecimiento personal, con una mejor comprensión de sí mismo en armonía con el grupo. Para lograr este propósito se aplicaron diferentes métodos como: Análisis - síntesis, inducción - deducción, sistematización de opiniones y valoraciones, entrevistas y triangulación de fuentes y métodos.

\begin{abstract}
Educators, with the responsibility of positively influencing the integral formation of youth, have before them the complex task of contributing to the development and personal growth of young people; they must have at their disposal alternatives that allow them to intentionally lead this complicated responsibility. It is the author's purpose to suggest ways, actions of educational orientation, procedures that contribute to enhance in youth the ability to assume their own personal growth, with a better understanding of themselves in harmony with the group. To achieve this purpose, different methods were applied such as: Analysis - synthesis, induction - deduction, systematization of opinions and assessments, interviews and triangulation of sources and methods.
\end{abstract}




\section{INTRODUCCIÓN}

La orientación educativa a los jóvenes por parte de sus educadores, entre los que ocupan un lugar importante los maestros y docentes en todos los niveles educativos, líderes sociales y comunitarios, padres, entre otros agentes educativos con responsabilidad de influir positivamente en su formación integral, debe estar orientada a lograr que estos se impliquen y comprometan consigo mismo a ser mejores, a ejercer un control activo y consciente de su actuación con vistas a su crecimiento personal. Para ello, se hace necesario capacitar a los educadores para que sepan orientarlos en el por qué, para qué y cómo lograrlo.

En este sentido, se aporta una propuesta de cómo proceder para facilitar el crecimiento personal de los jóvenes, a partir de indicadores, procedimientos, acciones de orientación educativa que, una vez concientizados, sirvan de pautas para guiar el camino a seguir en dirección a este objetivo.

Entre los métodos que sirvieron para hacer esta investigación se encuentran: Análisis - síntesis, inducción - deducción, sistematización, entrevistas y triangulación de fuentes y métodos.

\section{DESARROLLO}

Los educadores, no solo son los maestros que tienen el encargo social de educar a los niños, adolescentes y jóvenes, sino también los líderes sociales, políticos y comunitarios que surgen a partir del ejemplo personal por sus cualidades personales y personológicas, su activismo social, compromiso humanitario y aceptación de los miembros de la comunidad y sociedad en general. Estos, generalmente, ocupan responsabilidades sociales que le son asignadas por la selección y designación de las personas que lo conocen y pueden dar fe de su meritoria labor social.

En esta propuesta nos referimos a aquellos que, por su carisma, por su labor social y responsabilidad individual pueden influir educativamente en los jóvenes, y, que no siempre cuentan con las herramientas necesarias que le permitan mediar para contribuir a su crecimiento personal. Es propósito de la autora divulgar una propuesta válida que precisa a partir de indicadores claves cómo proceder para lograr tan importante empeño.

Los indicadores que se proponen como guía para promover el crecimiento personal de los jóvenes son:

1. La implicación movilizativa del joven para asumir su propio cambio. Significa una actitud activa en la satisfacción de sus necesidades y motivaciones más importantes, el buscar maneras objetivas y responsables para satisfacerse y saber representarse cambios, logros y éxitos personales, con el predominio de estados afectivos positivos.

2. El desarrollo de los autorreferentes con vistas a la formación del sí mismo. Implica la apropiación positiva de cualidades personales, saber cuáles son sus potencialidades y estimularlas, basados en la aceptación de su identidad personal, el autoconocimiento, autovaloración, autoaceptación, autoconfianza, seguridad propia, autoestima, autorregulación, autoexigencia, autodeterminación u otros.

3. Proyección hacia el futuro. Permite la adecuada proyección futura con vistas a su la realización personal, siendo necesario plantearse nuevas y objetivas metas, aspiraciones, planes o proyectos de vida a corto, mediano y largo plazos y sentirse confiado, seguro de lo que puede lograr, así como lograr ser capaz de tomar decisiones de acuerdo a sus posibilidades y mantenerlas para alcanzar sus propósitos.

4. El carácter positivo de las relaciones de convivencia. Facilita la atención y el respeto a los otros según sus particularidades personales, el liderazgo y las responsabilidades compartidas, la ayuda mutua, las relaciones afectivas positivas, la adecuada integración social al concebir y desarrollar objetivos grupales, el respeto a sí mismo y a los demás de acuerdo a sus potencialidades, aptitudes, posibilidades, con derecho a intervenir, decidir, aportar, aprender y asumir de manera reflexiva límites, reglas y normas de comportamiento y saber cumplirlas.

Como procedimientos importantes que pueden utilizarse para llevar a cabo la orientación educativa a los jóvenes se proponen: el diálogo, la reflexión y el autorreflexión sistemáticas basadas en estos indicadores.

El diálogo debe caracterizarse como una conversación amena y comprometida entre el educador y los jóvenes donde estos puedan exteriorizar, con sinceridad, confianza y respeto mutuo, sus formas de pensar, sus valoraciones, ideas o afectos, con el propósito de intercambiar actitudes, posiciones, estados, criterios sobre el mundo, la sociedad, las demás personas y sí mismo. En ese sentido, el diálogo, es también un encuentro o contacto que surge con el propósito de lograr un acuerdo que beneficie a ambas partes a favor de su bienestar como seres humanos con potencialidades para desarrollarse y crecer personalmente. El cual puede apoyarse o tener en cuenta los indicadores antes expuestos. 
Los procedimientos autorreflexivos se concretan en la reflexión sobre la acción y la reflexión durante las acciones que realiza el joven a través de las diversas actividades en que se inserta. En este sentido, el autorreflexión moviliza el potencial autorregulador del joven al reflexionar junto con él sobre:

- Qué indicadores pudiera tener en cuenta para potenciar su crecimiento personal y el de los demás. Por qué y para qué.

- Qué acciones puede desarrollar, en correspondencia con estos indicadores, para poder producir cambios necesarios en él y los demás.

- Cómo puede proceder para autoconocer, autoevaluar o autovalorar cómo va progresando personalmente, hasta dónde puede llegar y por qué.

- En quién o quiénes puede apoyarse para encontrar solución a sus problemas y ejecutar las acciones con mayor calidad

- Qué hacer si retrocede o abandona las acciones o tareas. Qué caminos, vías o alternativas puede seguir y sugerir a los demás.

En correspondencia con los indicadores y procedimientos propuestos se sugiere un conjunto de acciones de orientación educativa que pudieran servir de pautas a los educadores para concebir y ejecutar su labor de orientación a los jóvenes para estimularlos a aprender a crecer personalmente.

\section{Acciones para promover la implicación movilizativa del joven hacia su propio cambio}

- Para estimular la actitud activa en la satisfacción de sus necesidades y motivaciones más importante:

- Dialogar reflexivamente sobre la importancia de atender oportuna y de forma adecuada la satisfacción de sus necesidades más importantes (alimentación, afecto, descanso, sueño, vestimenta, movimiento, estudio, independencia, comunicación, de contacto social, de orientación profesional y sexual, sentido del deber, autoestima, otras), por qué y cómo satisfacerlas.

- Promover el desarrollo de la amplitud y diversidad de las motivaciones, aprovechando las potencialidades de la comunidad, de la escuela, la provincia estimulándolo a participar activa y protagónicamente en variadas actividades, fundamentalmente aquellas que tienen que ver con sus organizaciones juveniles; por ejemplo, la Federación de Estudiantes de la Enseñanza Media (FEEM) y la Federación Estudiantil Universitaria (FEU).

- Estimular sistemáticamente el enriquecimiento de su interés cognoscitivo en aquellas áreas del saber por las que demuestra preferencia o aptitud, a través de actividades, con énfasis en aquellas de carácter investigativo.

- Asistir oportunamente en el cómo se puede armonizar la satisfacción de necesidades individuales, con necesidades escolares, sociales, familiares, guiando en el cómo hacer una adecuada jerarquización de las mismas.

- Potenciar formas de conducta, estados afectivos y actitudes positivas ante las posibles frustraciones y conflictos, que estimulen la adopción de una actitud flexible y optimista donde prevalezca el buen humor.

- Para ayudar al joven a lograr un enfrentamiento positivo a sus principales problemas personales:

- Identificar los posibles problemas que le afectan. Existe una realidad y es que todo problema mal identificado no tiene solución; hay problemas que pueden ser planteados, que como tal no lo son, debido a cómo se perciben. Muchas veces el problema está en el joven mismo, en sus prejuicios, creencias, experiencias, en cómo lo percibe él o las personas más allegadas a él, que lo llevan a apreciar un problema determinado donde realmente no lo hay, o, a ver muy difícil una solución que depende, quizás, de un cambio en la manera de percibirlo.

- Jerarquizar adecuadamente los problemas identificados para poder ayudarlos a encontrarles posibles soluciones. No se puede pretender solucionar todos los problemas a la vez, se hace imprescindible ayudarlo a priorizar aquellos que más les afectan.

- Determinar las posibles causas que generan los problemas. Sólo con el conocimiento de las causas se puede trabajar mejor con las posibles soluciones, considerándolas para actuar sobre ellas.

- Analizar las posibles opciones, vías o alternativas de solución. Al investigar las causas, se puede facilitar este análisis, consciente de que las opciones, vías o alternativas no significan dar recetas o consejos como "camisas de fuerza" para ser aplicados inflexiblemente. Hay que considerar las posibilidades que posee el educando para asumirlas y el contexto donde estas se manifiestan. 
Existen problemas que pueden tener una solución, hay otros que pueden tener varias soluciones y otros que no la tienen, en este caso hay que ofrecer ayudas para aprender a convivir con ellos sin que afecte 0 , por lo menos, afecte lo menos posible su desarrollo y el crecimiento personal. También existen otros que no tienen una solución total y definitiva, sólo parcialmente, lo más aconsejable en este caso es facilitar opciones que provoquen mejoría. Para ello es necesaria la confianza en sí mismo, asumir un desempeño auténtico, creativo, se puede reformular o replantear opciones o el problema en sí mismo.

Por otra parte, hay soluciones que no dependen del joven, ni siquiera de sus familiares allegados. Se impone lo planteado anteriormente. La mejoría está en ayudarlo a ser flexible, positivo, optimista, indulgente, estar siempre por encima, avanzar de algún modo, con la experiencia, con el intento, al buscar opciones personales, cuando se establece el compromiso consigo mismo a ser mejor, a pesar de los problemas, lo cual puede lograrse al ocupar el tiempo libre en hacer cosas provechosas, que lo alejen de las preocupaciones que generan estos problemas: trazarse nuevos y alcanzables planes personales de vida, escuchar música, leer, observar un buen programa televisivo o una película e intercambiar puntos de vistas sobre ellos, fomentar una buena amistad, visitar amistades, pasear juntos, conversar, intercambiar gustos, poblar su mente de pensamientos positivos, observar detenidamente lo que le rodea buscando la belleza en lo que existe, sobre todo en la naturaleza, al decorar el espacio donde transcurre su vida (a través de una higiene adecuada, con afiches, fotografías, sembrar y cuidar plantas ornamentales, dibujar u otras opciones). Tratar siempre de sonreír, de dar y darse aliento, de tener confianza y seguridad propias.

- Establecer los compromisos mutuos para trabajar por la solución de sus problemas, el compromiso debe ser asumido durante todo el proceso de ayuda, para la solución del o los problemas, pero existen momentos en que se hace necesario reconsiderar el compromiso contraído y, si es necesario, asumir otros responsablemente.

\section{Acciones para orientar el desarrollo de autorreferentes con vistas a la formación del sí mismo:}

- Promover un interés sano por su apariencia personal, dándole apoyo, maneras alternativas de cómo lucir mejor: al estimular la limpieza física, el olor de la piel, el cabello lustroso, la cara y las uñas arregladas, los dientes y encías limpios que hacen la sonrisa más encantadora, proponer formas de vestir y peinados que destaque lo atractivo y minimicen defectos.

- Convocar formas de hacer ejercicios físicos para mantener la figura, la salud y el bienestar.

- Demostrar con anécdotas, hechos, películas, vídeos, cuentos u otras vías el por qué "la mejor y más bella apariencia física es la del "corazón", las cualidades personales positivas superan mucho las cualidades corporales y defectos físicos. El adorno más hermoso que puede llevar un joven es la vestidura incorruptible de una forma de ser bondadosa, amorosa, sincera, honesta, sencilla, modesta, fraterna, inteligente.

- Demostrar cómo el buen gusto en el vestir declara independencia, madurez y da un sello personal e individual a las personas. Cuando se viste presionado por lo que usan los demás, o por la moda, lo que se hace es mostrar dependencia de los demás.

- Promover la construcción de la identidad personal, al fomentar el sentimiento justo hacia su persona, su origen, aun cuando sea problemático y humilde, al valorar positivamente aquello que lo caracteriza o identifica y lo hace notable como miembro de una familia, grupo, patria, lo cual forma parte de su identidad. Es importante demostrar cuán justo es la asunción con dignidad de su propia identidad, como premisa para la apertura respetuosa a la identidad del otro.

- Promover la autoestima adecuada, estimulando la toma de conciencia de que la autoestima constituye el amor propio, el aprender a quererse a sí mismo, expresado en la satisfacción y conformidad personal con respecto a su físico, identidad, sentimientos, valores, cualidades. Se debe dialogar frecuentemente sobre el cómo puede fomentar el desarrollo de la autoestima y cómo saber distinguir la autoestima adecuada del amor propio inadecuado, ya sea por exceso (sobrestimación) o por defecto (subestimación), demostrando las repercusiones negativas para el crecimiento de la persona. Ha de ser convocado para que rechace en sí y en los demás cualquier manifestación o expresión que tengan que ver con el narcisismo, el egocentrismo, el individualismo, el egoísmo, la envidia.

Es importante, además, determinar las causas que pueden afectar el adecuado desarrollo de la autoestima. Por ejemplo, modelos educativos negativos desde el hogar, los continuos tropiezos con la realidad, la falta de experiencia, el no estar a la altura de lo que los otros esperan de él. Hay padres y profesores que hacen exigencias por encima de las posibilidades reales del joven; una nota sobresaliente que no se puede alcanzar sin valorar el esfuerzo que hace y la ansiedad que provoca en él no poder lograrlo, imponerle una profesión o una novia determinada, las comparaciones injustas entre un estudiante y otro, el menosprecio a su persona, el no ser tenido en cuenta. 
La crítica incesante o frecuente, también resulta una causal fatal para el adecuado desarrollo de la autoestima. A veces en el esfuerzo que se hace por corregir el mal, se tiende a la censura o al castigo constante. Debe tenerse en cuenta que la crítica, si se repite incesantemente, aturde, pero no reforma. Una atmósfera de crítica hostil es fatal para sus esfuerzos. Ocurre que el joven a quien se censura frecuentemente por alguna falta, la llega a considerar como peculiaridad suya, algo contra lo cual es en vano luchar, y así se da origen al desaliento, a la desesperación, genera más indisciplina, o una aparente impotencia, indiferencia o fanfarronería. La acción fundamental que facilitaría el desarrollo de la autoestima es brindar amor, propiciar que este sentimiento de algún modo sea fomentado entre todos.

\section{Acciones para estimular la adecuada proyección personal futura:}

- Promover la comprensión de que la adecuada proyección personal significa la disposición a enfrentar nuevas tareas, metas, retos, a esbozar proyectos o planes de vida en correspondencia con sus condiciones y potencialidades, así como también de formar y desarrollar la capacidad para saber reorientarse ante situaciones difíciles, imprevistas o cambiantes (cambio de grupo, mudanza, pérdida o ausencia de un familiar querido, otros), de no cejar ante el empeño de alcanzar lo que se propone, sus aspiraciones y proyectos, aunque haya que enfrentar dificultades y además aconsejar formas constructivas en su relación con otras personas.

- Estimular a través del elogio o recompensa merecida los éxitos o avances en la consecución de sus propósitos o proyectos, premisas para su realización personal, induciéndolo a sentirse satisfecho consigo mismo por los resultados que vaya alcanzando poco a poco.

\section{Acciones dirigidas a desarrollar el carácter positivo de las relaciones de convivencia:}

- Influir en la comunidad y en el joven para ser tolerante y comprensivo con las demás personas.

- Explicar cuán dañino resultan los ataques a los demás, ya sea de palabras o hechos.

- Mediar ecuánimemente ante cualquier arrebato de cólera o impulsividad e instar a la disculpa cuando se hicieran actos que afectaran el bienestar o estima del otro.

- Dar fe de cómo en el grupo todos pueden crecer, mejorar, aprender, vencer.

- Aprovechar los errores que se cometan como premisas para el mejoramiento humano.

- Acompañar a cada uno hacia el alcance de logros y éxitos, sin crear dependencias, ni actitudes posesivas, ni superficiales.

- Compulsar el aprendizaje de derechos personales y a decir frases como "yo puedo", "yo valgo", "tengo una respuesta", "escúchenme", "no puedo ahora, quizás después", "respeten mi opinión", "yo siento" y otras.

- Promover máximas personales tales como: "aprender a escuchar", "aprender a crecer", "aprender a ser feliz", "lograr ser uno mismo", "viva la amistad", "haz con los demás lo que tú deseas que hagan contigo", otros.

- Promover la sinceridad, el comprometimiento y el entendimiento mutuo como una forma de respetar las diferencias.

- Estimular la reflexión sobre por qué es imposible pretender una sola forma de pensar, de ser, de actuar en las relaciones de convivencia y por qué las diferencias no tienen que ser irreconciliables.

- Reafirmar el valor del respeto. Apoyarse en la frase "Respetar para que te respeten", cada miembro debe aprender a interpretar deberes y derechos, a seguir reglas de interacción de manera consciente, lo que implica disciplina y la determinación de límites para la actuación.

- Compulsar el cumplimiento de derechos, tratando de convencer que los derechos son parámetros que se establecen para cumplir con ética el comportamiento, que admiten reflexión sistemática y están ligados a deberes. Cada miembro debe aprender a identificar su posición de legalidad y lealtad frente a los otros.

- Practicar la tolerancia, que significa aprender a vivir respetando estilos y personalidades, no significa de ninguna manera "aguantar", aunque se prive de derechos, ni tampoco aceptar una excesiva permisividad. Se hace necesario comprender que actuar en tolerancia, es estar dispuesto a la concesión y a la transacción atendiendo a lo que se puede y a lo que no se puede. Hay momentos, espacios, actitudes con los que hay que ser intolerantes ante el chantaje, la deslealtad, la traición y otros. Se debe exigir ser tolerante sobre la base de premisas aprobadas entre todos.

- Ofrecer opciones de cómo aprender a disfrutar y a ser feliz, sobre todo al aconsejar vivir con amor, este es un sentimiento que no puede ser sustituido por otros, como los celos, el odio, la envidia, la ambición, la calumnia, estos conllevan inevitablemente a la infelicidad personal. El disfrutar y ser feliz se logra en la cotidianeidad de cada día provechoso que se vive con alegría, cuando se aprende a apreciar lo que se tiene, a recrear la realidad, a saber disfrutar del buen humor, al apreciar el gozo que provoca la 
autotransformación positiva y cada instante placentero y considerarlo como ascenso en la vida, así como disfrutar también el ascenso y el bienestar de los demás, caracterizándose por ser positivo y al hacer cada día alguna acción, de la que la persona pueda sentirse orgullosa y satisfecha de sí misma.

- Colaborar en la superación de conflictos o contradicciones en las relaciones personales. Ello ayudaría a enfrentar los problemas en las relaciones interpersonales, para lo cual es importante enseñar a conversar, que significa entender la importancia que tiene el respetar las opiniones ajenas, aprender a escuchar, a autocontrolarse, a dar respuestas y recibirlas sin gritar, vociferar, gesticular.

Es intención que la propuesta realizada se divulgue por diferentes vías, a través de la superación, preparación e impresión de folletos, materiales y artículos dirigidos a los agentes y líderes comunitarios para ser introducida en su accionar cotidiano, al tiempo que contribuyan a la validación de la efectividad de la misma con vistas a su continuo perfeccionamiento.

\section{CONCLUSIÓN}

La orientación educativa de los educadores hacia los jóvenes con los cuales entran en relación directa, es muy importante para estimular su crecimiento personal. Se propones métodos, procedimientos y acciones que bien orientados pueden contribuir a estimular la actitud activa en la satisfacción de sus necesidades y motivaciones más importantes; a lograr un enfrentamiento positivo a sus principales problemas personales; el desarrollo de autorreferentes con vistas a la formación del sí mismo; su adecuada proyección personal futura y a desarrollar el carácter positivo de las relaciones de convivencia. Se hace necesario implicarlos y movilizarlos a favor de su desarrollo y crecimiento a través del diálogo, la reflexión y el autorreflexión sistemáticas en un clima psicológico favorable donde la comunicación y el estímulo oportuno los motiven y estimulen optimistamente.

\section{BIBLIOGRAFÍA}

1. Caballero, AN. 2006. Situación del desarrollo en la juventud. Material impreso. Santiago de Cuba.

2. Collazo, B y Puentes. M. 2002. Orientación en la actividad pedagógica. Ed. Pueblo y Educación, Ciudad de la Habana.

3. Colectivo de autores. 2002. Psicología para educadores. Ed. Pueblo y Educación. Ciudad de La Habana.

4. Colectivo de autores. 2007. Materiales básicos del Módulo II. Psicología para educadores y Diagnóstico individual y grupal, orientación y prevención en el contexto escolar.

5. Cubela G., J. M. (2015). Propuesta metodológica para el diseño, evaluación y control de los proyectos educativos de año o grupo. Ponencia presentada en 1. Congreso Internacional de Ciencias Pedagógica en el Instituto Superior Tecnológico Bolivariano de Tecnología. Guayaquil. Ecuador. Del 26 al 28 de marzo. ISBN 978-9942-17-011-8.

6. Cubela G., J. M. (2016). Labor Educativa con los Jóvenes un Reto de las Universidades en el Siglo XXI. Vol. 4 Núm. 1 (2016): Septiembre - Diciembre Revista Investigación, Formación y Desarrollo: Generando Productividad Institucional, ISSN 1390-9789 LATINDEX 2651, DOI: https://doi.org/10.34070/rif.v7i. Ecuador.

7. Cubela, J. M. (2018). La labor educativa con la juventud en el Siglo XXI. Desafíos, proyecciones y experiencias en la educación universitaria. Editorial Académica Española is in cooperation with: MoreBooks! Marketing SRLBalti, 4 Industriala street, Moldova, Europe. Link: https://www.eae-publishing.com/extern/listprojects. (Consultado 11 de junio de 2019).

8. Cubela, J. M. (2018). Caracterización, diagnóstico, orientación educativa y crecimiento personal de los jóvenes: Orientaciones, vías, métodos, técnicas y ejemplificaciones para su realización. Editorial Academic Espanola is in cooperation with: MoreBooks! Marketing SRL Balti, 4 Industriala Street, Moldova, Europe. Link: https://www.eae-blishing.com/extern/listprojects (Consultado 5 de julio de 2019).

9. Cubela, J. M. (2018). Desafíos, resultados y satisfacción social de la proyección educativa en la Universidad de Oriente. Cuba. Publicado en las memorias de Congress proceedings con ISBN 978-9942-17-033-0. Ecuador. IV Congreso Internacional de Ciencias Pedagógicas. Ecuador. 